\title{
DE LA DISTINCTION AFFECTIF/INTELLECTUEL À UNE THÉORIE DE LA PHRASE MODAliséE. RePRÉSENTATIONS MENTALES ET opérations linguistiques chez Charles Bally
}

\author{
Anamaria Curea \\ Université Babeş-Bolyai, Cluj-Napoca, Roumanie
}

\section{Résumé}

Dans l'histoire des théories énonciatives du $\mathrm{xx}^{\mathrm{e}}$ siècle, Charles Bally est l'auteur de la première «théorie générale de l'énonciation », bien que son nom ait été rarement évoqué par les théoriciens de ce champ. Dans le cadre de la réflexion sur l'articulation entre représentations et opérations chez plusieurs linguistes de l'énonciation, dans un rapport plus ou moins étroit avec une image saussurienne, nous envisageons d'éclairer les points les plus révélateurs qui ont conduit Bally de la stylistique à la théorie de l'énonciation, en poursuivant un fil qui va de la distinction entre «impressions》 et «idées pures» (Précis 1905) à l'opposition affectif/intellectuel (Traité 1909), puis au traitement de l'expressivité linguistique en tant que mécanisme ( $L e$ langage et la vie 1926) pour arriver à la théorie de l'énonciation, où il définit la modalité. Dans ce parcours, nous évoquons son interprétation originale de concepts et de conceptualités saussuriennes, ainsi que la position de Saussure à l'égard du statut de l'affectif dans la linguistique.

\section{Mots-clés}

Bally, Saussure, stylistique, théorie de l'énonciation, affectif/intellectuel, modalité

\section{Abstract}

In the history of the enunciative theories of the twentieth century, Charles Bally is the author of the first "general theory of enunciation", although his name has rarely been mentioned by the theorists of the field. In the context of the study of the relations between representations and operations among several enunciation theorists, in a more or less close relationship with the image of Saussure, we plan to shed light on the most revealing points that led Bally from stylistics to the theory of enunciation, pursuing a thread that goes from the distinction between "impressions" and "idées pures" (Précis 1905) to the affective / intellectual opposition (Traité 1909), then to the treatment of expressiveness as a mechanism (Le langage et la vie 1926), to arrive at the theory of enunciation, where he defines modality. In this course, we discuss also his original interpretation of Saussurean concepts and conceptualizations, as well as Saussure's position on the status of the affectivity in linguistics.

\section{Keywords}

Bally, Saussure, stylistics, theory of enunciation, affective/intellectual, modality

Dans l'histoire des théories de l'énonciation du $\mathrm{xx}^{\mathrm{e}}$ siècle et malgré les mentions relativement rares par les théoriciens de ce champ, le nom de Charles Bally occupe une place importante, en tant qu'auteur d'une première «théorie générale de l'énonciation », titre de la première section de son ouvrage de 1932, Linguistique générale et linguistique française. Comme ce fut le cas aussi pour Albert 
Sechehaye, avec qui il a œuvré pour faire connaître la pensée de Ferdinand de Saussure, en tant qu'éditeurs (et défenseurs) du Cours de linguistique générale, il a développé un programme scientifique et construit des conceptualités qui ne sont ni un «ajout» ni un «prolongement» de l'œuvre du maître (Puech 2008, p. 1086), mais plutôt un laboratoire où les idées saussuriennes ont été réinscrites et réinterprétées de manière originale dans une théorie nouvelle.

Dans le cadre de la réflexion sur l'articulation entre représentations et opérations chez plusieurs linguistes de l'énonciation, dans un rapport plus ou moins étroit avec une image saussurienne, nous envisageons d'éclairer les points qui nous semblent révélateurs pour le parcours théorique de Charles Bally depuis la stylistique jusqu'à la théorie de l'énonciation. Sa stylistique n'est pas considérée uniquement comme l'une des contributions les plus importantes à l'histoire de la discipline - un programme fondateur, bien que sans postérité en tant que tel-, mais aussi comme une approche linguistique singulière de l'affectivité ${ }^{1}$.

Cette problématique a été développée sous diverses formes durant toute son activité scientifique. Selon le principe chronologique, nous constatons que l'approche linguistique de l'affectivité a un caractère évolutif dans la théorie du linguiste genevois. Nous poursuivrons ainsi un fil conceptuel qui va de la distinction entre « impressions » et «idées pures » (Précis de stylistique française 1905, ci-après $P S F$ ) à l'opposition affectif/intellectuel (Traité de stylistique française, 1909, ci-après $T S F$ ), puis au traitement de l'expressivité linguistique en tant que mécanisme (Le langage et la vie 1926, ci-après $L V$ ) pour arriver à la théorie de l'énonciation, où il introduit la distinction entre dictum et modus, représentation et assertion modalisée (Linguistique générale et linguistique française 1932, ci-après $L G L F$ ).

Dans la dernière partie de cette réflexion nous tenons à évoquer l'opinion de F. de Saussure à l'égard du programme de Bally, qui, bien qu'exprimée brièvement et dans des textes à caractère privé ou institutionnel (lettres adressées à son collègue, rapport sur la création d'une chaire de stylistique), est néanmoins révélatrice des différences conceptuelles qui caractérisent son programme et celui de Charles Bally.

\section{Le PRÉCIS DE STYLISTIQUE FRANÇAISE (1905): «IMPRESSIONS》 ET « IDÉES PURES»}

Le premier ouvrage publié par Charles Bally sous le nom de stylistique fixe les principes de recherche qui guident bon nombre de ses publications ultérieures, et tente d'instituer et d'autonomiser un nouvel ordre de recherche, une nouvelle

1 Saluée par Joseph Vendryes (1946-1947, p. 57), qui présente son collègue genevois comme un novateur. Pour une analyse de leur correspondance qui atteste en même temps leurs affinités personnelles et les rapports entre l'école de Genève et l'école de Paris, voir Testenoire (2015 et 2017). Sur l'école de Genève, son identité, ses rapports avec d'autres écoles linguistiques, voir C. Puech 2015 (dir.). 
méthode et un nouvel objet pour la linguistique. Il s'agit d'identifier, de classer et de caractériser les «moyens d'expression» ou les «procédés généraux » dont dispose une langue pour « rendre par la parole les phénomènes du monde extérieur aussi bien que les idées, les sentiments et en général tous les mouvements de notre vie intérieure » (Bally 1904, p. 7). Cette première définition de la stylistique est fondée sur l'extension maximale de la notion d'expression, résumant à la fois la parole comme activité (champ de manifestation du rapport entre la pensée et la langue, ayant pour finalité la représentation du monde objectif et de la sphère de la subjectivité) et la parole dans une langue donnée. Au croisement de ces deux acceptions de la parole se situe le domaine de la stylistique.

Par «moyens d'expression », Bally entend l'ensemble des opérations linguistiques diversifiées, autant lexicales et grammaticales que mimo-gestuelles, mais susceptibles d'être classées et analysées (les groupements synthétiques, la forme de la phrase, le mode du verbe, l'ellipse, le tour interrogatif, le langage exclamatif, etc.), par lesquelles les représentations mentales du sujet parlant (les «impressions » et les « idées pures ») se réalisent dans l'usage « réel » et « actuel » de la langue. L'objet de la stylistique a essentiellement une dimension discursive, et l'activité langagière est préférentiellement envisagée comme manifestation expressive de deux classes d'éléments : une classe ou un type intellectuel (sous la forme des « idées pures ») et un type affectif, pas encore théorisé clairement, qui résume les «sens impressifs» des mots et des constructions. En affirmant la nécessité de prendre en considération le contexte, d'analyser l'emploi réel et actuel des mots, d'étudier les sens impressifs ou intuitifs des mots et des expressions, Bally propose les premières formulations de la distinction (à caractère méthodologique) entre les modes d'expression intellectuel et affectif sur laquelle est fondée le TSF de 1909.

Dans le $P S F$, deux enjeux sont à l'origine du programme stylistique : la nécessité d'orienter les études linguistiques vers l'usage, la «langue parlée», sans pour autant faire acquérir à l'objet linguistique une dimension individuelle, et conjointement, la nécessité de prendre en compte l'affectif dans sa dimension linguistique. Le premier enjeu prend la forme d'un programme de linguistique synchronique, fondé sur un impératif systémique qui rappelle la conceptualité saussurienne $^{2}$, mais qui est orienté vers un ensemble de données linguistiques différentes. Le deuxième enjeu prend la forme de la recherche d'un principe de classement de données linguistiques hétérogènes, spécifiques à un corpus diversifié, fluctuant et soumis à plusieurs types de variations (psycholinguistiques et sociolinguistiques notamment). Dans ce contexte spécifique, la problématique de l'affectif est construite à partir de l'importance attribuée aux sens impressifs des

2 Sur le développement des idées saussuriennes chez Charles Bally et Albert Sechehaye, voir Amacker (2000) et Godel (1984). 
faits de langue dans l'usage courant, réel et actuel. La stylistique présuppose une «connaissance intuitive » des mots, et en même temps, une correspondance et une homologie entre les représentations de l'esprit et leur expression linguistique. C'est cette connaissance impressive des mots qui permet «que ceux-ci éveillent des représentations dans notre esprit et inversement, qu'une représentation trouve aisément les mots qui la rendent au mieux » (Bally 1905, p. 15). Le rapport établi dans le $P S F$ entre la présence ou l'absence de nuances impressives (effets sur la sensibilité et l'imagination des sujets parlants) et la prise en considération de leur réception par l'intelligence des sujets entendants est une première formulation, à notre sens, de la distinction entre les éléments intellectuels et les éléments affectifs du langage sur laquelle est fondée la deuxième définition de la stylistique, qui figure dans le $T S F$.

La stylistique est à la recherche d'une unité qui soit conforme à son objet, mais cette recherche n'est pas aisée. « Moyen d'expression » correspond à une acception générale de cette unité, à une définition compréhensive et cela correspond aux deux enjeux du programme stylistique. Le mot comme unité de délimitation suscite de fortes réserves, étant donné qu'il ne correspond pas à une « unité psychologique »; la base d'observation idéale ou la norme étant la langue parlée, l'hypothèse d'une correspondance entre «l'idée exprimée» et «le symbole linguistique» qui l'exprime oriente vers une unité à caractère synthétique, soit des phrases, soit des «groupements synthétiques» :

Nous pensons par phrases et non par mots, et le triage des éléments s'opère inconsciemment en nous sur ces matériaux réunis en faisceaux. Le mot est une unité lexicologique et non une unité psychologique (Bally 1905, p. 31)

L'impératif de la contextualisation, des effets produits, est un prisme qui, d'un côté, ouvre la voie vers un nouveau type d'unité conforme au nouveau type de données, et, d'un autre côté, invite à se manifester, à l'intérieur de la linguistique, des points de vue nouveaux sur langage, venant notamment de la psychologie et aussi de la sociologie ${ }^{3}$. Les définitions de la périphrase et du langage figuré caractérisent bien cette approche que Bally qualifie d' «embryonnaire» :

Pour bien comprendre la périphrase, il faudrait y voir une opération de l'esprit beaucoup plus qu'un fait d'expression. Si l'on en élargit la notion, elle apparaît comme le mouvement même de la pensée, au lieu d'être un procédé plus ou moins artificiel; pour cela il ne faut pas la resserrer dans des cadres fixes (ibid., p. 94)

3 Le «métissage des points de vue, sociologique et psychologique, tout particulièrement, sur le langage » est l'une des acceptions de la généralité en linguistique (Fournier, Colombat, Puech 2010, p. 212). Sur la linguistique sociologique de Charles Bally, voir Forel (2008). 
La plupart des hommes pensent par impressions, les idées pures ne les satisfont pas; le plus souvent la sensibilité est mêlée à l'élaboration des idées; l'expression de ces idées porte le même caractère, et c'est pour cela que les images sont nécessaires [...]. Ce que la stylistique veut connaître en étudiant les figures, comme tout le reste, c'est la distinction entre les formations libres, spontanées, et celles qui portent la marque de l'usage établi; elle veut reconnaître jusqu'à quel point une figure est sentie comme telle et dans quelle mesure elle a évolué vers l'abstraction; car c'est de là que dépend le sens plus ou moins impressif de l'image et c'est ce qui la classe comme moyen d'expression (ibid., p. 102)

Quant à l'analyse de la construction de la phrase dans la perspective stylistique, Bally a tendance à exclure les relations logiques entre l'expression et l'idée, et à privilégier l'action du sentiment sur l'ordre habituel des mots, justifiée par la nécessité psychologique. La grammaire et la syntaxe (habituelles) relèvent de la logique, qui est inexpressive, et la stylistique -toujours liée à une forme de psychologie- doit étudier l'effet du sentiment sur l'ordre logique. Idée et sentiment, grammaire et stylistique, logique et psychologie commencent à former des paires conceptuelles et progressivement, le deuxième terme de ces paires commence à l'emporter sur le premier dans la pensée de Bally, qui prend une tournure qualifiée d' « anti-logiciste », d' « anti-intellectualiste », « dans une matrice où la psychologie constitue la référence» (Chiss 1997 [1986], p. 160) :

Cette conception [la phrase comme énoncé d'un jugement, notre ajout] du langage ne peut se maintenir devant l'observation des faits; elle est en désaccord avec la constitution de l'esprit humain. Chez la plupart des hommes la sensibilité, si l'on prend ce mot dans son sens le plus large, prime l'intelligence ; le langage ne peut être avant tout une opération intellectuelle (Bally 1905, p. 127)

Bally envisage ainsi deux classes d'opérations linguistiques, qui sont homologues aux deux types de représentations mentales: le langage subjectif ou affectif est opposé au langage objectif, et assimilé au langage appelé sentimental. Cette seconde forme de langage a deux caractéristiques: elle se superpose au langage discursif ou langage des idées, car l'idée est énoncée lexicalement, alors que le sentiment est exprimé par les procédés du langage sentimental, à savoir l'intonation, la mimique, les gestes, et elle précède le langage des idées, dans la mesure où elle n'est pas encore affranchie des sensations, la forme la plus pure du langage subjectif étant le langage enfantin, presque entièrement affectif:

Nous parlons simultanément deux langues, et c'est parce que nous n'en observons généralement qu'une, la plus facile à saisir, la langue objective, que tant de malentendus règnent encore sur la nature du langage. [...] Le sentiment s'exprime dans une langue à lui, il a des moyens d'expression qui lui appartiennent en propre; son vocabulaire et sa syntaxe ne sont pas ceux de la langue objective. Mais voici qui est bien plus paradoxal: pour exprimer les 
sentiments, il n'a pas même besoin de la voix; en second lieu, il peut se servir de la voix sans articuler des mots; et enfin, s'il fait usage des mots, c'est pour leur donner une signification qu'ils n'ont pas par eux-mêmes (Bally 1905, p. 130131).

Selon Bally, l'étude de la langue parlée amène à la conclusion que la parole sert à extérioriser prioritairement des impressions et des sentiments plutôt que des idées, et à renforcer l'idée que « la partie subjective ou affective du langage doit avoir sa place, peut-être même la première, dans la recherche des moyens d'expression, objet de la stylistique » (ibid., p. 130). L'originalité de la stylistique est là : étudier la «vie du langage» là où elle se manifeste, dans la relation interlocutive et dans la sphère de l'affectivité qui lui correspond.

\section{Les «MODES D’EXPRESSION» AFFECTIF ET INTELLECTUEL DANS LE TRAITÉ DE STYLISTIQUE FRANÇAISE (1909)}

Les principes posés dans le $P S F$ sont repris dans le $T S F$, ouvrage ample en deux tomes, aux visées théorique et didactique. À l'origine de la stylistique comme discipline scientifique se trouve l'hypothèse qui envisage la possibilité (et aussi la nécessité) de distinguer, dans la langue parlée notamment, mais sans exclure la langue écrite ou la langue littéraire, entre les éléments intellectuels et les éléments affectifs. Ces classes d'éléments forment deux modes d'expression qui ne sont pas séparés dans la réalité, mais se combinent en diverses proportions ${ }^{4}$ :

Les faits d'expression reposent sur des combinaisons psychologiques où chaque élément entre dans des proportions variables; la bonne méthode consiste à dégager dans chaque cas le facteur dominant d'après lequel un phénomène stylistique peut être classé [...]. Cette recherche peut être secondée par des connaissances élémentaires de psychologie, de même que le côté social du langage est plus facile à saisir si l'on possède quelques notions sur les tendances qui régissent les sociétés. Mais en étudiant ces sciences auxiliaires, il faut bien se garder de les faire passer au premier plan, car leur rôle est pour nous secondaire; nous ne faisons pas de psychologie du langage, pas plus que nous ne prétendons faire de la sociologie. Toute notre attention se porte sur la face expressive et non sur la face intérieure des faits de langage: le langage est ici but, non moyen (Bally 1909, p. 28).

Cette conception dialectique n'empêche pas la stabilisation de l'objet de la stylistique en fonction du contenu affectif des faits d'expression. La définition donnée dans le $T S F$ diffère considérablement de celle qui est proposée dans le PSF :

4 L'attention portée au caractère relatif de la distinction affectif-intellectuel est probablement une réponse à des critiques exprimées par F. de Saussure et A. Sechehaye à ce propos. 
La stylistique étudie donc les faits d'expression du langage organisés au point de vue de leur contenu affectif, c'est-à-dire l'expression des faits de la sensibilité par le langage et l'action des faits de langage sur la sensibilité (Bally 1951 [1909], p. 16).

L'affectif devient un critère de sélection et de classement des données linguistiques qui font l'objet de la stylistique. La méthode stylistique vise à dégager les caractères affectifs des faits d'expression, répartis en deux classes - caractères affectifs naturels et effets par évocation-par comparaison avec des termes d'identification recherchés dans la langue normale ou intellectuelle, pour la première classe, et dans la langue commune, pour la seconde classe. L'affectif est délimité en fonction d'un élément de contraste, l'intellectuel, et infuse le métalangage de la nouvelle discipline-éléments affectifs, valeur affective, dominante affective, côté affectif, contenu affectif, mode d'expression affectif ${ }^{5}$ :

On sait en effet qu'il n'y a jamais d'expression entièrement intellectuelle ou entièrement affective, parce que l'intelligence et le sentiment, ces deux aspects (je ne dis pas ces deux facultés) de notre esprit, se trouvent mêlés à toutes nos pensées; donc, seule, la proportion importe. Étant donné un fait de langage quelconque, il faut déterminer quel est l'élément qui prédomine dans la conscience de ce fait et lui donne sa marque propre. Le dosage est-il tel que l'expression apparaît essentiellement intellectuelle ou essentiellement affective? Voilà, ramené à sa formule la plus générale, l'objet de la stylistique (Bally 1951 [1909], p. 157-158).

Cette approche poursuit le projet défini dans le PSF, et notamment une forme d'homologie entre les représentations mentales et les opérations linguistiques, mais en essayant de trouver et d'appliquer une méthode permettant une différenciation et un classement, fussent-ils généraux, des procédés linguistiques identifiables en fonction de leur contenu affectif:

Un principe important de notre méthode, c'est l'établissement, par abstraction, de certains modes d'expression idéaux et normaux; ils n'existent nulle part à l'état pur dans le langage, mais ils n'en deviennent pas moins des réalités tangibles, dès qu'on observe 1. Les tendances constantes de l'esprit humain et 2 . les conditions générales de la communication de la pensée (Bally 1951 [1909], p. 29).

5 L'ambiguïté de la notion d'affectivité chez Charles Bally est attribuée par Sylvie Durrer à une oscillation dans son emploi : elle renvoie tantôt à un mode d'expression, tantôt à toute attitude énonciative. Deuxièmement, tout en considérant les deux pôles comme également pertinents, il privilégie pourtant le mode affectif, parce qu'il est «le plus fréquent», «le plus naturel » et «le plus vivant» des modes d'expression (Durrer 1998, p. 115). Du point de vue chronologique, nous constatons que le changement intervient là où l'affectif cesse d'être considéré comme un critère de classement et de description d'un fait de langue pour acquérir une dimension explicative de la dynamique de la langue, autrement dit dans une théorie du langage. 
Les deux sciences auxiliaires mentionnées par Bally, les points de vue psychologique et sociologique, sont impliquées dans l'identification de ces modes d'expression idéaux. Le premier contribue à définir un mode d'expression intellectuel ou logique ou langage des idées pures, auquel il faut mesurer les différences de sens entre les faits d'expression ainsi que la présence ou l'absence de caractère affectif; le deuxième est impliqué dans la définition de la langue commune, en fonction de laquelle on mesure les "faits d'évocation de milieu» (ibid., p. 29).

\section{LE «MÉCANISME DE L’EXPRESSIVITÉ LINGUISTIQUE», UN NOUVEAU DISPOSITIF THÉORIQUE}

L'article intitulé «Le mécanisme de l'expressivité linguistique » figurant dans la seconde édition de 1926 du recueil $L V$, est un texte auquel aussi bien Sechehaye (1927) que Meillet (1927) ont attribué, au moment de sa publication, un statut théorique important. Ce texte n'a pas seulement une visée descriptive, mais offre les principaux éléments d'un système explicatif cohérent de la dynamique langagière, qui rassemble des éléments théoriques ayant déjà été exposés dans des textes antérieurs. Dans ce nouveau cadre, sous l'influence de son vitalisme ${ }^{6}$, l'affectif n'est plus un critère de sélection et de classement des données, tout en étant associé, comme dans la stylistique, au langage de la vie et de l'action, ainsi qu'à l'expression du sentiment et de l'émotion du sujet parlant.

Deux éléments marquent ce changement de visée : la définition du mécanisme de l'expressivité linguistique et le rapport d'antinomie envisagé entre la communication et l'expression ${ }^{7}$. Ces deux définitions intègrent des concepts saussuriens en les réinscrivant dans une approche qui continue, en principe, le programme stylistique.

Le mécanisme de l'expressivité linguistique est résumé comme suit:

Le langage, intellectuel dans sa racine, ne peut traduire l'émotion qu'en la transposant par le jeu d'associations implicites. Les signes de la langue étant arbitraires dans leur forme - leur signifiant - et dans leur valeur, - leur signifié- les associations s'attachent soit au signifiant, de manière à en faire jaillir une impression sensorielle, soit au signifié, de manière à transformer le concept en représentation imaginative. L'une et l'autre catégorie d'associations se chargent d'expressivité dans la mesure où la perception sensorielle ou la représentation imaginative concorde avec le contenu émotif de la pensée (Bally 1977 [1926], p. 83).

Les concepts saussuriens d'arbitraire, de forme, de valeur, de signifiant et de signifié sont utilisés de manière originale pour induire l'idée que l'expressivité

6 L'impact des idées de Bergson sur la conception de Bally dans $L V$ (1913 et éditions ultérieures) et $L G L F$ a été analysé par Médina 1985, p. 95-104.

7 Pour le développement de la problématique de l'expression et de l'expressivité chez Ch. Bally, A. Sechehaye et H. Frei de 1900 à 1940, voir Curea (2015). 
s'oppose à l'arbitraire, par des associations sur le signifiant et sur le signifié qui sont des formes de motivation. Les associations sur le signifiant produisent, par leur combinaison avec des signifiants arbitraires, des perceptions correspondantes aux idées, et, par là-même, des impressions sensorielles. C'est le cas des interjections, mais aussi des phrases exclamatives, des combinaisons de voyelles et de consonnes (gazouiller, caracoler, grignoter, cliquetis), des contrastes de timbres (zigzaguer), de l'accent d'insistance (« une fformidable explosion»), de la quantité longue ou brève des syllabes, de répétitions de voyelles (tohu-bohu) ou de consonnes (papoter, barboter), de syllabes (dada, nounou), de mots («C'est loin, loin», «un père est toujours un père»), de pauses entre les syllabes («refus ca-té-go-rique), entre les mots (je-le-veux) et de tous les procédés rythmiques.

Les associations sur le signifié visent à transformer le concept en représentation imaginative: c'est un cas d'expressivité par substitution. Dans cette catégorie Bally classe les images, les figures ou les tropes (un clou dans la dent, c'est une forte tête, la ville est en rumeur, ses yeux lancent des éclairs, le vent mugit). Bally parle d'hypostases (empruntant le terme à d'autres linguistes), définies comme des substitutions d'une catégorie à une autre (au lieu de «Que vous êtes naïf!», on dit «Que vous êtes enfant!»-le substantif enfant ayant une fonction adjective), en généralisant cette notion, synonyme pour lui de transposition d'une catégorie dans une autre.

Le deuxième élément de ce système explicatif est le rapport d'antinomie envisagé entre l'expression et la communication, qui deviennent les pôles d'une échelle :

La pensée tend vers l'expression intégrale, personnelle, affective; la langue cherche à communiquer la pensée vite et clairement: elle ne peut donc la rendre que dans ses traits généraux, en la dépersonnalisant, en l'objectivant. Plus les échanges se multiplient, plus la communication travaille à l'encontre de l'expression personnelle. [...] Quand la langue arrive à ses fins, le signe linguistique devient purement conventionnel, ou, comme dit de Saussure, arbitraire : et il l'est non seulement dans sa forme matérielle et phonique-son signifiant, - il l'est aussi dans sa signification, sa valeur - son signifié (Bally 1977 [1926], p. 80).

L'affectivité se situe au pôle de l'expression, en association avec d'autres notions, l'illogisme, la pensée, le langage, la motivation, les innovations personnelles, la subjectivité. S'opposant au pôle de l'expression, celui de la communication organise un autre réseau notionnel, où se retrouvent l'objectivité, la langue, le social, la logique, l'arbitraire ou le conventionnel. Dans $L V$, par la place qui lui est assignée par rapport à l'antinomie de l'expression et de la communication, l'affectivité reçoit une valeur explicative des innovations individuelles et de leur importance dans la dynamique de la langue et le 
changement linguistique. Si dans la stylistique, l'affectif apparaît comme un attribut proprement linguistique, intégré à une théorie essentiellement descriptive d'une langue donnée, dans $L V$ il est un concept intégré à une théorie du langage, à une théorie explicative de la dynamique de la langue et acquiert une valeur argumentative. Si dans la formulation du mécanisme, Bally réutilise des concepts saussuriens, dans la formulation de l'antinomie se retrouve une conceptualité saussurienne, intégrée d'une manière tout aussi originale que les concepts.

\section{LinguistiQue GÉNÉRALE ET LINGUISTIQUE FRANÇAISE:}

PHRASE, MODALITÉ, ÉNONCIATION

Historiquement, $L G L F$ de 1932 est le premier ouvrage qui propose une «théorie générale de l'énonciation », en ouverture de la première partie intitulée Principes de linguistique générale. Cet ouvrage se distingue parmi les autres du même auteur par la présence d'éléments appartenant à un nouveau système conceptuel. Parmi ces éléments, l'un des plus importants est celui de modalité. Son importance se mesure à un autre changement révélateur: le sujet de la stylistique, sujet pensant, est de plus en plus doublé ou même remplacé par un véritable sujet parlant, grâce à l'attention accordée aux opérations linguistiques, qui ne sont plus envisagées comme homologues des représentations mentales (les cas exemplaires sont le mensonge, l'ironie et la fiction littéraire).

Ici l'affectif est subordonné à la notion de modalité. Cette réflexion ciblée sur la phrase comme représentation modalisée, sur le modus et le dictum dans la phrase, fixe les jalons principaux vers une prise en considération du sujet comme un je énonciateur, qui se distingue de la représentation du sujet comme siège des sentiments, des émotions et des volitions. Cette nouvelle conception ne s'inscrit pas contre la première, mais la dépasse en l'intégrant dans un cadre qui définit et étudie l'énonciation, et non seulement le contenu affectif des faits d'expression.

Dans $L G L F$, l'opération linguistique (l'ordre du linguistique) est définie comme distincte de la représentation (l'ordre du mental ou du psychique), dans le cadre d'une approche théorique de la phrase modalisée :

Penser, c'est réagir à une représentation en la constatant, en l'appréciant ou en la désirant.

C'est donc juger qu'une chose est ou n'est pas, ou estimer qu'elle est désirable ou indésirable, ou enfin désirer qu'elle soit ou ne soit pas. On croit qu'il pleut ou on ne le croit pas, ou on en doute, on se réjouit qu'il pleuve ou on le regrette, on souhaite qu'il pleuve ou qu'il ne pleuve pas.

Dans le premier cas, on énonce un jugement de fait, dans le second un jugement de valeur, dans le troisième une volition. 
La première opération relève de l'entendement, la deuxième du sentiment, la troisième de la volonté, qui a son aboutissement dans l'action, aboutissement qui est une des fonctions du langage tout en le dépassant.

La pensée ne se ramène donc pas à la représentation pure et simple, en l'absence de toute participation active d'un sujet pensant (Bally 1965 [1932], p. 35).

Dans cette première partie d'une théorie générale de l'énonciation, Bally définit la modalité, après avoir distingué entre le dictum et le modus :

La phrase explicite comprend donc deux parties : l'une est le corrélatif du procès qui constitue la représentation (p. ex. la pluie, une guérison); nous l'appellerons, à l'exemple des logiciens, le dictum.

L'autre contient la pièce maîtresse de la phrase, celle sans laquelle il n'y a pas de phrase, à savoir l'expression de la modalité, corrélative à l'opération du sujet pensant. La modalité a pour expression logique et analytique unverbe modal (p. ex. croire, se réjouir, souhaiter), et son sujet, le sujet modal; tous deux constituent le modus, complémentaire du dictum.

La modalité est l'âme de la phrase; de même que la pensée, elle est constituée essentiellement par l'opération active du sujet parlant. On ne peut donc pas attribuer la valeur de phrase à une énonciation tant qu'on n'y a pas découvert l'expression, quelle qu'elle soit, de la modalité. (ibid., p. 36)

La modalité est donc indispensable à la définition de la phrase, car elle est nécessaire à son fonctionnement. C'est l'occasion, pour Bally, de proposer deux conceptualisations qui ont permis des ouvertures ultérieures, l'une vers la théorie polyphonique et l'autre vers les développements ultérieurs de la problématique des modalités $^{8}$. La première concerne la situation où, malgré l'identité entre sujet modal et sujet parlant, il faut distinguer entre pensée personnelle et pensée communiquée: l'ironie, le mensonge et la fiction littéraire :

Mais même lorsque le sujet pensant est identique au sujet parlant, il faut prendre garde de confondre pensée personnelle et pensée communiquée. Cette distinction est de la plus haute importance, et s'explique par la nature et la fonction du signe linguistique lui-même. En effet, le sujet peut énoncer une pensée qu'il donne pour sienne bien qu'elle lui soit étrangère. Il s'agit alors d'un véritable dédoublement de la personnalité. Le mensonge en est la forme la plus caractéristique [...]. Cela nous amène à une conception particulière de la réalité en matière de sémiologie: le signe porte en lui-même sa signification (son signifié), et c'est celle-là seule qui compte pour la communication. Elle peut être en contradiction avec la pensée de celui qui emploie le signe, et ne recouvre donc pas la notion de réalité. En dehors du langage, c'est la raison d'être de la fiction littéraire et poétique : on peut dire que les aventures de don Quichotte sont autant de mensonges de Cervantès (Bally 1965 [1932], p. 37).

La deuxième concerne les formes de réalisation de la modalité qui vont de l'explicite à l'implicite :

8 Pour une analyse du caractère extensif de l'énonciation chez Bally, voir Chiss (1997). 
1. Je veux (j'exige) que vous sortiez.

2. Je vous ordonne (vous intime l'ordre) de sortir.

3. Il faut que vous sortiez.

4. Vous devez sortir.

5. Sortez!

6. À la porte!

7. Ouste!

8. Geste indiquant la porte et jeu de physionomie marquant une volonté irritée.

9. Expulsion pure et simple du perturbateur.

Bally signale que la phrase 2) ne contient pas le pronom-sujet vous; dans 3), le sujet modal reste imprécis, le verbe modal étant impersonnel ; 4) exprime le modus d'une façon indirecte, car devoir n'est pas un verbe modal, mais un auxiliaire de mode; 5), une des formes les plus usuelles, ne renferme aucun mot de nature modale ; 6) réduit la phrase à un seul terme nominal qui fait place dans 7) à une exclamation, et dans 8) à une mimique; quant à 9) (expulsion du perturbateur), il s'agit d'un procédé évidemment extérieur au langage, mais qui montre l'étroite parenté de celui-ci avec l'action.

La modalité, qui est corrélative de l'énonciation, peut se réaliser sur le mode explicite, mais aussi par les moyens suivants :

a. «éléments non articulatoires de la langue » : l'intensité, l'intonation, la durée, les pauses ou silences, le rythme;

b. interjections, dont les valeurs sont diverses et nuancées, autant modales que dictales (1. Interjections qui expriment des émotions et des volitions (des exclamations); elles sont de nature modale (Eh $e h !=«$ Voilà qui est assez curieux », Chut !=Taisez-vous!) 2. Interjections descriptives (onomatopées), qui sont dictales (Boum, Patatras $=$ Quelque chose est tombé). 3. Interjections à fonction de «signaux», déictiques (Pst!, Holà $!=« C$ 'est à vous que je m'adresse!»-elles expriment des rapports et rappellent les ligaments grammaticaux

c. la mimique et la gestuelle: notons que l'expression des émotions et des volitions est renvoyée à la catégorie du modal. La même catégorie comprend également des signes de nature non verbale : les gestes sont classés de même que les interjections, car ce sont des équivalents de phrase. Il y a ainsi des gestes « exclamatifs» et modaux (un haussement d'épaules peut signifier « cela m'est indifférent», froncement de sourcils et regard perçant «telle et telle chose me met en colère »); des formes onomatopéiques (inviter quelqu'un à s'approcher par un geste déictique approprié), « dans les langues normalement constituées, le geste a le plus souvent une fonction subalterne et se borne à accompagner la parole» (ibid., p. 43). 
d. «signes situationnels»: contexte actuel ou «circonstances connues des interlocuteurs», autrement dit, un savoir partagé antérieur au moment de l'énonciation.

Rapportée à la problématique de l'intonation, la catégorie de l'affectif glisse également vers le «modal», sans que cette transition terminologique soit explicitement signalée :

Dans nos langues, elle (l'intonation) est un procédé essentiellement syntaxique, c'est-à-dire autonome, réalisable uniquement dans la parole, et applicable à un nombre théoriquement illimité d'énonciations. Sa valeur est modale, autrement dit, elle exprime ou souligne la modalité sans laquelle il n'y a pas de phrase; elle actualise la phrase comme la pensée actualise la représentation. Dans tout cela, rien qui rappelle la motivation des mots. Mais les choses changent lorsqu'une expression modale a une valeur unique ou un nombre déterminé, limité de valeurs; dans ce cas, l'intonation motive dans la mesure où cette limitation est perceptible (Bally 1965 [1932], p. 131-132, nous soulignons).

Nous constatons donc, pour ce qui est de l'intonation, des signes extraarticulatoires, ou des signes non verbaux, que l'expression des émotions et des volitions, qualifiée d' « expressive» dans les travaux de stylistique, est associée à la catégorie de la modalité, aux valeurs modales des faits linguistiques. Ce choix est anticipé par des considérations de même nature lors d'une conférence prononcée en 1930 sur la «crise du français » (La Grammaire), où la distinction entre jugement de fait et jugement de valeur conduit Bally à définir la phrase comme «accouplement d'un fait avec une appréciation» (p. 83):

Une représentation pure et simple ne constitue jamais une phrase; celle-ci implique une adhésion du sujet parlant, qui la juge vraie ou fausse, la désire ou la craint, la veut, en commande la réalisation à un autre ou l'interdit, etc. Or, cette partie subjective de la phrase, quelle est son expression grammaticale ? C'est la modalité, c'est-à-dire l'ensemble des procédés par lesquels la langue indique les réactions du sujet parlant vis-à-vis de ses représentations (Bally 2004 [1931], p. 84, nous soulignons).

La subjectivité est située à un cadre différent, celui de l'énonciation. La perspective est moins psychologisante, orientée vers le pôle proprement linguistique.

\section{Ferdinand de Saussure et le programme de Bally}

Des documents à caractère personnel ou institutionnel attestent que Ferdinand de Saussure connaissait bien les travaux de stylistique de Bally, qu'il a évoqués assez brièvement trois fois, mais chaque fois de manière révélatrice sur les différences de conception entre lui et son collègue : deux lettres de Saussure à Bally - lettre du 3 juillet 1904, relative à un manuscrit sur la stylistique, lettre de septembre 1912, 
relative au projet de création d'une chaire de Psychologie du langage, en concurrence avec celle de Linguistique générale - et le rapport de Saussure sur la création d'une chaire de Stylistique à l'université de Genève.

Dans la première lettre, reproduite dans l'article «Correspondance BallySaussure» du CFS 481994 (1995), p. 91-134, on lit les propos suivants :

Lorsque vous arrivez à la définition de la stylistique [...] je me suis demandé si quelques lecteurs ne trouveraient pas un peu abrupt d'apprendre que celle-ci doit consister finalement dans l'étude de ce qui est affectif dans le langage. [...] Mais on n'était pas préparé dans un autre sens : en effet, $\left[\mathrm{w}^{\circ}\right]$ et si parfaits que soient les paragraphes préliminaires sur la différence de nature entre l'intellectuel et l'affectif, je n'en ai pas trouvé qui fût destiné à marquer leur importance comparative, de fait et non de principe et par conséquent à fixer pour l'affectif telle ou telle étendue dans l'ensemble des phénomènes. Sauf erreur, celui qui aurait cette idée préconçue que l'affectif existe, mais est complètement insignifiant \{dans la somme de\} ses manifestations, n'en serait pas détourné, - ou pas formellement détourné - par la lecture de vos chapitres préliminaires à la définition qui fera une chose capitale de cet affectif. C'est le point qui m'a frappé, et dont je devais en conscience vous faire part. Il me semble que c'est positivement une lacune (tout un chaînon manque), mais en même temps ce n'est qu'une lacune. Un ou deux paragraphes intercalés, et tout devient clair.

La critique majeure formulée par Saussure concerne l'importance comparative de l'intellectuel et de l'affectif dans l'analyse, ce qui touche au cœur même de la stylistique. La pertinence de la démarche est mise en question, selon Saussure, par l'impossibilité de fixer une étendue de l'affectif dans l'ensemble des phénomènes (l'affectif existe-t-il formellement?). Le problème que pose la stylistique n'est pas d'envisager la distinction de principe entre l'intellectuel et l'affectif dans l'expression, mais de vouloir fixer une étendue pour l'affectif dans les manifestations langagières. L'aspect le plus problématique que présente la stylistique de ce point de vue est l'impossibilité de fixer pour les caractères affectifs une existence à part, en tant qu'éléments analysables en eux-mêmes. En utilisant le mot étendue, Saussure rend compte d'un fait essentiel : il aura compris la stylistique de Bally comme un projet qui se propose d'assigner une forme à des éléments qui ne peuvent aspirer à un tel statut (en dissociant et en délimitant des faits psychiques qui sont très imbriqués), car l'affectif n'existe pas formellement. S'il a une existence, ce dont Saussure ne doute pas d'ailleurs, celle-ci est d'une nature différente. Cela explique, nous semble-t-il, l'oscillation permanente de Bally entre la linguistique et la psychologie.

Dans une lettre adressée à Bally en septembre 1912, relative au projet de création d'une chaire de Psychologie du langage, en concurrence avec celle de Linguistique générale, Saussure mentionne le psychologisme de l'approche stylistique de Bally : 
La stylistique, telle que vous l'avez conçue, érige en méthode constante cette observation [1'observation psychologique] et la poursuit spécialement dans les nuances du discours. Elle serait une «psychologie du discours» si vous ne montriez, par une vue féconde, que dans le discours s'élabore, en fait, ce qui devient ensuite partie intégrante du langage» (lettre de Saussure à Bally, reproduite dans le recueil Sur la stylistique, éd. É. Karabétian, 2007, p. 43).

Selon Saussure, la méthode stylistique dépasse l'observation psychologique des «nuances du discours» par une approche dynamique interne du système linguistique et $\mathrm{du}$ mode d'existence très particulier qui est propre à la langue. Les remarques de Saussure sur la stylistique de Bally sont en même temps des indices très éclairants, à notre avis, sur la manière dont il conçoit le rapport dialectique entre langue et parole.

Et enfin, dans son Rapport sur la création d'une chaire de stylistique datant de 1912, on peut lire :

Messieurs, j'en arrive à dire ce qu'on pourrait véritablement craindre comme danger à propos de la chaire de stylistique, ce n'est pas du tout les préventions dérivant de l'équivoque avec science du style, mais au contraire l'objection consistant à dire : mais alors, c'est tout simplement de la linguistique qu'on nous offre sous le nom de stylistique. Oui, messieurs, tout simplement de la linguistique. Seulement, la linguistique, j'ose le dire, est vaste. Notamment elle comporte deux parties : l'une qui est plus près de la langue, dépôt passif, l'autre qui est plus près de la parole, force active et origine véritable des phénomènes qui s'aperçoivent ensuite peu à peu dans l'autre moitié du langage. Ce n'est pas trop que les deux [ ].

En résumé : $1^{\circ}$ non ce qui est individuel mais ce qui est consacré par l'usage social, remplissant ainsi les conditions qui font qu'une chose est linguistique : $2^{\circ}$ non nécessairement ce qui est écrit mais de préférence ce qui est parlé; $3^{\circ}$ non dans un but normatif et pour donner les règles de la bonne expression, mais

$4^{\circ}$ enfin, avec le but de généraliser les observations, d'arriver à une théorie applicable aux langues.

Bien loin qu'il [ ], l'objection réelle qui pourrait se présenter, c'est qu'on dise : c'est tout simplement alors de la linguistique. En effet; seulement le domaine de la linguistique est vaste; elle a suffisamment à faire, j'ose le dire, dans les compartiments plus élémentaires, ou bien dirigés dans d'autres sens, comme par exemple l'histoire évolutive des formes. Par conséquent, c'est une chose bienvenue, [ ] l'explication des formules de la langue en tant que motivées par tel ou tel état psychologique» (Saussure 2002, p. 272-273).

Le seul problème signalé par Saussure est autant de type terminologique que de type institutionnel : la stylistique de Bally, malgré un rapprochement hâtif avec la science du style, est tout simplement de la linguistique et la chaire de stylistique risquerait ainsi de semer la confusion parmi ses auditeurs, en prétendant qu'on leur présente une théorie stylistique, qui ne serait pas essentiellement une linguistique. Saussure défend pourtant la raison d'être de la nouvelle discipline, évoquant un des 
aspects essentiels présentés dans son cours de linguistique générale, à savoir la dualité langue/parole. Il faut remarquer que même dans ce texte, la distinction ne semble pas avoir un caractère si tranchant que dans certains passages du $C L G$ : « Notamment elle comporte deux parties : l'une qui est plus près de la langue, dépôt passif, l'autre qui est plus près de la parole, force active et origine véritable des phénomènes qui s'aperçoivent ensuite peu à peu dans l'autre moitié du langage. Ce n'est pas trop que les deux [ ]». La distinction entre linguistique de la langue et linguistique de la parole, telle qu'elle a été présentée dans le $C L G$ ne doit pas être conçue dans l'absolu, la linguistique pouvant se rapprocher tantôt de l'une, tantôt de l'autre, sans faire tort à aucun des deux volets de la théorie. Le rôle de la parole résulte clairement de sa définition en termes de force et d'origine: elle est la condition d'existence de la langue et son principe générateur.

Toute linguistique est psychologique «à un degré quelconque». Mais pour que l'étude du discours ne devienne pas essentiellement psychologique, le linguiste doit situer correctement le sujet parlant par rapport à l'activité de parole et à la langue proprement dite. La notion de discours est utilisée trois fois par Saussure relativement à l'approche stylistique de Bally. Étudier le système des moyens d'expression d'une langue est un programme centré sur l'activité discursive et sur son rôle dans la dynamique de la langue. Ainsi Saussure serait-il le premier à considérer la stylistique de Bally comme une approche discursive et signaler le danger de tomber dans un psychologisme qui risque d'oblitérer son intérêt linguistique. Étudier le discours du point de vue des états affectifs qui motivent tel ou tel emploi d'un moyen d'expression a sa place dans la linguistique à condition de rendre compte de la dynamique interne de la langue, dont le discours est le reflet.

\section{POUR CONCLURE}

À l'étape initiale de son œuvre, Charles Bally envisageait sous plusieurs formes une adéquation ou une homologie entre la pensée et la langue qui faisait coïncider les représentations mentales du sujet parlant (« intellectuelles » et « affectives ») avec les opérations linguistiques (les «modes d'expression» intellectuel et affectif). Dans la définition du mécanisme de l'expressivité linguistique, l'affectif cesse d'être un critère de contrôle des données, et acquiert une dimension explicative des innovations et de la dynamique de la langue. Les opérations linguistiques sont envisagées de manière plus autonome par rapport aux représentations mentales du sujet parlant.

Dans son dernier ouvrage, la modalité, inséparable de l'énonciation, marque une étape où les opérations linguistiques sont définies et analysées en dehors de l'idée d'une homologie avec les représentations mentales. Dans la théorie de la phrase, la modalité est clairement envisagée comme une opération linguistique proprement dite, sans interférence terminologique ou conceptuelle avec l'ordre des représentations mentales. 


\section{BIBLIOGRAPHIE}

\section{Sources primaires}

Bally, Charles, 1905. Précis de stylistique: esquisse d'une méthode fondée sur l'étude du français moderne, Genève, Eggimann.

- 1951 [1909]. Traité de Stylistique française, 2 vol., Genève, Librairie Georg, Paris, Klincksieck.

- 1977 [1926]. Le langage et la vie, $3^{\mathrm{e}}$ édition augmentée, Genève, Droz.

— 2004 [1931]. La crise du français. Notre langue maternelle à l'école, édité par Jean-Paul Bronckart, Jean-Louis Chiss et Christian Puech, Genève-Paris, Droz.

- 1965 [1932]. Linguistique générale et linguistique française, $4^{\mathrm{e}}$ édition, Berne, Francke.

- 2007. Sur la stylistique : articles et conférences, édité, présenté, annoté et commenté par Étienne Karabétian, Paris, Eurédit.

«Correspondance Bally-Saussure », CFS 481994 (1995), 91-134.

\section{Sources secondaires}

Amacker, René, 2000. «Le développement des idées saussuriennes chez Bally et Sechehaye », Historiographia linguistica 27, 205-264.

Chiss, Jean-Louis, 1997 [1986]. "Charles Bally: qu'est-ce qu'une “théorie de l'énonciation"? », Jean-Louis Chiss et Christian Puech, Fondations de la linguistique. Études d'histoire et d'épistémologie, $2^{\mathrm{e}}$ édition, Bruxelles, Duculot, 159-167.

Colombat, Bernard, Fournier, Jean-Marie, Puech, Christian, 2010. Histoire des idées sur le langage et les langues, Paris, Klincksieck.

Curea, Anamaria, 2015. Entre expression et expressivité : l'école linguistique de Genève de 1900 à 1940. Charles Bally, Albert Sechehaye, Henri Frei, Lyon, ENS Éditions.

Durrer, Sylvie, 1998. Introduction à la linguistique de Charles Bally, Lausanne-Paris, Delachaux et Niestlé.

Forel, Claire-Antonella, 2008. La linguistique sociologique de Charles Bally. Étude des inédits, Genève, Droz.

Godel, Robert, 1984 [1961]. "L'école saussurienne de Genève», Cahiers Ferdinand de Saussure 38, 77-82.

Meillet, Antoine, 1927. «Le langage et la vie », $2^{\mathrm{e}}$ édition augmentée (Paris, Payot, 1926), Bulletin de la Société de linguistique de Paris 82, 14-16.

Médina, José, 1985. "Charles Bally: de Bergson à Saussure», Langages 77, 95-104.

Puech, Christian, 2008. "Qu'est-ce que faire l'histoire du "récent"?», Actes du Congrès mondial de linguistique française, Paris, Institut de langue française, 1081-1090.

- 2015 (dir.), «Faire école en linguistique au $x^{\mathrm{e}}$ siècle : l'école de Genève», Histoire Épistémologie Langage 37/2.

Vendryes, Joseph, 1946-1947. «L'œuvre linguistique de Charles Bally», Cahiers Ferdinand de Saussure 6, 48-62.

Testenoire, Pierre-Yves, 2015. «L'école de Genève vue de la Société de linguistique de Paris », Histoire Épistémologie Langage 37/2, 53-70.

- 2017. "À l'ombre de deux maîtres: la correspondance Bally-Vendryes », Valentina Chepiga et Estanislao Sofia (dir.), La correspondance entre linguistes. Un espace de travail, 45-63.

Saussure, Ferdinand de, 2002. Écrits de linguistique générale, édité par Simon Bouquet et Rudolf Engler, Paris, Gallimard.

Sechehaye, Albert, 1927. "L'école genevoise de linguistique générale », Indogermanische Forschungen 44, 217-241. 